\title{
ARBITRARINESS OF THE GENERAL SOLUTION AND SYMMETRIES
}

\author{
WERNER M. SEILER \\ Institut für Algorithmen und Kognitive Systeme \\ Universität Karlsruhe, D-76128 Karlsruhe, Germany \\ Email:kg04@ibm3090.rz.uni-karlsruhe.d400.de
}

\begin{abstract}
A bstract. The computation of the number of arbitrary functions in the general solution is briefly reviewed. The results are used to study normal systems and their symmetry reduction. We discuss the treatment of gauge systems, especially the analysis of gauge fixing conditions. As examples the Yang-Mills equations with the Lorentz gauge and Einstein's vacuum field equations with harmonic coordinates are considered.
\end{abstract}

\section{Introduction}

If one can not determine the general solution of an equation, as it is usually the case with differential equations, one wants to know at least the dimension of the solution space. This is, however, not trivial for systems, especially if they are overdetermined. It turns out, that involution [10] provides the key. We showed in a recent paper [13], how to compute the number of arbitrary functions and their arity for closed representations of the general solution of an involutive system.

The purpose of this paper is to provide more applications of these results. Normal systems are the natural generalization of single equations with respect to the arbitrariness. Symmetry reductions $[1,7]$ are most often performed for such systems. We will compute in Section 4 the loss of generality during such a reduction.

Gauge systems are a central theme in modern theoretical physics. We will present in Section 5 a compared with [13] improved treatment of them through the introduction of gauge corrected Cartan characters. Einstein [2] pioneered the analysis of the arbitrariness of field theories. His philosophy was to choose by otherwise equal properties the system which restricts the fields stronger.

Special emphasis will be put on the analysis of gauge fixing conditions. As concrete examples, the last two sections treat the Yang-Mills equations and Einstein's vacuum field equations in arbitrary dimensions. For the former we will analyse the Lorentz gauge, for the latter harmonic coordinates.

\section{Formal Theory}

Formal theory [10] is based on jet bundle formalism. Let $x_{1}, \ldots, x_{n}$ and $u^{1}, \ldots, u^{m}$ be a local coordinate system on a bundle $\mathcal{E}$. We define a differential equation of order $q$ as a fibred submanifold $\mathcal{R}_{q}$ in the jet bundle $J_{q} \mathcal{E}$. Locally, $\mathcal{R}_{q}$ is given by a system of equations $\Phi^{\tau}\left(x^{i}, u^{\alpha}, p_{\mu}^{\alpha}\right)=0$, where $p_{\mu}^{\alpha}=\partial^{\mu_{1}+\cdots+\mu_{n}} u^{\alpha} / \partial\left(x^{1}\right)^{\mu_{1}} \cdots \partial\left(x^{n}\right)^{\mu_{n}}$ for the multi-index $\mu=\left[\mu_{1}, \ldots, \mu_{n}\right]$. 
The prolongation $\mathcal{R}_{q+1} \subset J_{q+1} \mathcal{E}$ is obtained by formally differentiating all equations with respect to the independent variables $x^{i}$. It is well-known that during prolongation integrability conditions can arise. They can occur at any prolongation order. A system that does not generate integrability conditions is called formally integrable, because it is possible to construct order by order a formal power series solution.

A formally integrable system is involutive, if it has an involutive symbol. The symbol $\mathcal{M}_{q}$ of $\mathcal{R}_{q}$ is a system of linear (algebraic, not differential!) equations in some unknowns $v_{\mu}^{\alpha}$ defined by

$$
\mathcal{M}_{q}: \sum_{\alpha,|\mu|=q} \frac{\partial \Phi^{\tau}}{\partial p_{\mu}^{\alpha}} v_{\mu}^{\alpha}=0 .
$$

For a quasi-linear equation the symbol is essentially obtained by substituting $v_{\mu}^{\alpha}$ for $p_{\mu}^{\alpha}$ in the highest-order part of the equation.

A jet variable $p_{\mu}^{\alpha}$ is said to be of class $k$, if $\mu_{k}$ is the first non-vanishing entry of the multi-index. We order the columns of the symbol $\mathcal{M}_{q}$ by class (highest class first) and compute a row echelon form. If $v_{\mu}^{\alpha}$ is the leading term of an equation in this solved form of the symbol, then $p_{\mu}^{\alpha}$ is called a principal derivative [3]. All other derivatives of order $q$ are parametric.

We define $\beta_{q}^{(k)}$ as the number of principal derivatives of class $k$. The symbol $\mathcal{M}_{q}$ is involutive, if

$$
\operatorname{rank} \mathcal{M}_{q+1}=\sum_{k=1}^{n} k \beta_{q}^{(k)}
$$

Associating with each equation whose principal derivative is of class $k$ its multiplicative variables $x^{1}, \ldots, x^{k}$, we see that if we prolong each equation with respect to its multiplicative variables only, we get algebraically independent equations. Equation (2) tells us, that in the case of an involutive symbol no further independent equations exist.

The Cartan characters

$$
\alpha_{q}^{(k)}=m\left(\begin{array}{c}
q+n-k-1 \\
q-1
\end{array}\right)-\beta_{q}^{(k)}, \quad k=1, \ldots, n
$$

count the parametric derivatives of order $q$ and class $k$. The Cartan-Kähler theorem states that for analytic involutive systems there exists a unique analytic solution for a certain initial value problem prescribing $\alpha_{q}^{(k)}$ analytic functions of $x^{1}, \ldots, x^{k}$ for $k=1, \ldots, n$ as Cauchy data. This generalizes the Cauchy-Kowalevsky theorem.

Although these definitions appear to be coordinate dependent, one can show that with the exception of certain singular systems every coordinate system yields the same values for the $\beta_{q}^{(k)}$. For lack of space, we can not discuss here the delicate question of $\delta$-regularity of a coordinate system but refer to the literature [10].

Finally, one should note that any system can be algorithmically completed to an involutive one [10,12]. This is ensured by the Cartan-Kuranishi theorem. Thus it poses no real restriction, if we assume from now on that we deal only with involutive systems. 


\section{Arbitrary Functions}

In a recent paper [13], we have shown how one can derive the number of arbitrary functions in the general solution of an involutive system. We briefly repeat the results here. The Cartan characters are the key tool for this analysis.

The parametric derivatives represent the arbitrariness in the general solution allowed by the differential equation, whereas the principal derivatives can be computed through the differential equation or its prolongations. The number of parametric derivatives of order $q+r$ is given by the Hilbert polynomial of the equation

$$
H_{q}(r)=\sum_{i=0}^{n-1}\left(\sum_{k=i}^{n-1} \frac{\alpha_{q}^{(k+1)}}{k !} s_{k-i}^{(k)}(0)\right) r^{i} .
$$

Here we have used the symmetric q-product

$$
s_{k}^{(n)}(q)=\sigma_{k}^{(n)}(q+1, q+2, \ldots, q+n) \text { for } 0 \leq k \leq n,
$$

where $\sigma_{k}^{(n)}$ denotes the elementary symmetric polynomial of degree $k$ in $n$ unknowns $\left(\sigma_{0}^{(n)}=1\right)$.

We assume now that the general solution of $\mathcal{R}_{q}$ can be written in a closed form containing some arbitrary functions and that its Taylor series expansion can be constructed order by order, i.e. there exists a bijection between its coefficients of order $q+r$ and the Taylor coefficients of order $q+r+j$ of the arbitrary functions of differentiation order $j^{1}$. Let there be $f_{k}, j$ such functions with $k$ arguments.

If we write the Hilbert polynomial as $H_{q}(r)=\sum h_{i} r^{i}$, we can derive the following linear diophantine system for the $f_{k, j}[13]$

$$
\sum_{j \in J} \sum_{k=i}^{n-1} \frac{n !}{k !} s_{k-i}^{(k)}(q-j) f_{k, j}=n ! h_{i}, \quad i=0, \ldots, n-1,
$$

where $J$ denotes the set of considered values for $j$ (usually $J \subset\{-q, \ldots, 0\}$ ). In general, this system has no unique solution, if $|J|>1$. Setting $J=\left\{j_{0}\right\}$ we obtain the following theorem.

Theorem 1 If there exists a representation of the general solution whose power series can be constructed order by order and whose arbitrary functions are all of differentiation order $j_{0} \geq-q$, then it contains $f_{k}$ arbitrary functions with $k$ arguments, where the $f_{k}$ are determined by the recursion relation

$$
\begin{gathered}
f_{n}=\alpha_{q}^{(n)} \\
f_{k}=\alpha_{q}^{(k)}+\sum_{i=k+1}^{n} \frac{(k-1) !}{(i-1) !}\left(\alpha_{q}^{(i)} s_{i-k}^{(i-1)}(0)-f_{i} s_{i-k}^{(i-1)}\left(q+j_{0}\right)\right) .
\end{gathered}
$$

Such a representation can only exist, if the solution of this recursion relation contains no negative integers.

\footnotetext{
1 This means that the function appears for $j<0$ as integrand of a $j$-dimensional integral and $j>0$ as a derivative of $j$-th order.
} 
(7) has a simple solution for $j_{0}=-q$, namely $f_{k}=\alpha_{q}^{(k)}$. This corresponds to the representation of the general solution given by the above mentioned Cartan-Kähler theorem. This means, that the arbitrary functions in the Cauchy data will in general enter as $q$-dimensional integrals.

Einstein [2] introduced as a simpler measure for the arbitrariness the strength of a differential equation. If we expand the rational function

$$
Z_{q}(r)=H_{q}(r) /\left(\begin{array}{c}
n+q+r-1 \\
n-1
\end{array}\right)
$$

in powers of $1 / r$, the strength $Z_{q}^{(1)}$ is defined as the coefficient of $1 / r$. It is related to the Cartan characters [13]

$$
Z_{q}^{(1)}=(n-1)\left(\frac{1}{2} n \alpha_{q}^{(n)}+\alpha_{q}^{(n-1)}\right)
$$

\section{Normal Systems}

Normal systems satisfy the conditions of the Cauchy-Kowalevsky theorem [4]. With a coordinate transformation a normal system of order $q$ can always be brought into the following solved form

$$
p_{[0, \ldots, q]}^{\beta}=\Phi^{\beta}\left(x^{i}, u^{\alpha}, p_{\mu}^{\alpha}\right), \quad \beta=1, \ldots, m
$$

where the right hand sides do not contain any derivative of order $q$ which is purely with respect to $x^{n}$.

In the terms introduced in Section 2, we see that (10) is solved for the derivatives of class $n$. Since we have as many equations as dependent functions, all such derivatives appear. Thus we find $\beta_{q}^{(n)}=m, \beta_{q}^{(n-1)}=\cdots=\beta_{q}^{(1)}=0$ and

$$
\alpha_{N}^{(n)}=0, \alpha_{N}^{(n-k)}=m\left(\begin{array}{c}
q+k-1 \\
q-1
\end{array}\right) .
$$

Theorem 1 with $j_{0}=0$ yields $f_{n}=0, f_{n-1}=m q$ and $f_{n-2}=m q(1-q) / 2$. Thus we have proven the following perhaps surprising result:

Theorem 2 An algebraic representation, i.e. one without integrals or derivatives, of the general solution of a normal system, whose power series can be constructed order by order, can exist only, if either there are only two independent variables or the system is of first order.

In the case of a first order system, the recursion relation (7) can be solved in closed form for $j_{0}=0$ [13]: $f_{n}=\alpha_{1}^{(n)}, f_{k}=\alpha_{1}^{(k)}-\alpha_{1}^{(k+1)}$. For a normal system, this yields the well-known values of the Cauchy-Kowalevsky theorem: $f_{n-1}=m$, all other $f_{k}$ vanish.

A standard technique for the construction of solutions is the reduction with respect to a symmetry group $[1,7]$. It is well-known that only for ordinary differential equations one can reconstruct the general solution of the original system from the 
general solution of the reduced system. In the case of partial differential equations, one obtains only special solutions.

Let us assume that the symmetry group operates with $s$-dimensional orbits and that after the reduction the system is still normal. Then we get a system with $\bar{n}=n-s$ independent and $\bar{m}=m$ dependent variables of order $\bar{q}=q-\Delta q$. The Cartan characters of the reduced system are $\bar{\alpha}_{\bar{q}}^{(\bar{n})}=0, \bar{\alpha}_{\bar{q}}^{(\bar{n}-k)}=m\left(\begin{array}{c}\bar{q}+k-1 \\ \bar{q}-1\end{array}\right)$. Thus if $\Delta q=0$, then $\bar{\alpha}_{q}^{(k)}=\alpha_{q}^{(k+s)}$. If $\Delta q>0$, we get an additional loss of generality as to expect.

Since the Cartan characters form a descending sequence [10]

$$
\alpha_{q}^{(1)} \geq \alpha_{q}^{(2)} \geq \cdots \geq \alpha_{q}^{(n)}
$$

this result means that we do not only loose all arbitrary functions with more than $n-s$ arguments, but that even the number of arbitrary functions with less arguments decreases.

Even in more general situations the loss of generality can be computed without explicit construction of the reduced system. One must simply compare the Cartan characters of the original system alone with the characters of the system plus the invariant surface condition (after completion to an involutive system!). An extension of this method to reductions with respect to generalized or to weak symmetries $[7,8]$ is straight-forward.

For instance, the analysis of Lie-Bäcklund symmetries of first-order, normal systems [6] leads to the addition of differential constraints of order $r$

$$
\Psi^{\tau}\left(x^{i}, u^{\alpha}, p_{\mu}^{\alpha}\right)=0, \quad\left\{\begin{array}{l}
\tau=1, \ldots, p, \\
|\mu| \leq r, \\
\mu_{n}=0 .
\end{array}\right.
$$

Note that $x^{n}$ occurs here only as parameter, because all derivatives with respect to $x^{n}$ can be eliminated using (10).

We assume that (13) is in involution and that there are $\tilde{\beta}_{r}^{(k)}$ equations of class $k$ for $k=1, \ldots, n-1$. The combined system $(10,13)$ has an involutive symbol, if and only if there exist matrices $\left(M^{i}\right)_{\rho}^{\tau}$ such that

$$
\left(M^{i}\right)_{\rho}^{\tau} \frac{\partial \Psi^{\rho}}{\partial p_{\mu}^{\alpha}}=-\frac{\partial \Psi^{\tau}}{\partial p_{\mu}^{\beta}} \frac{\partial \Phi^{\beta}}{\partial p_{i}^{\alpha}}, \quad\left\{\begin{array}{l}
i=1, \ldots, n-1, \\
|\mu|=r
\end{array}\right.
$$

No integrability conditions arise, i.e. the system is involutive, if and only if

$$
D_{n} \Psi^{\tau}-\sum_{\alpha,|\mu|=r} \frac{\partial \Psi^{\tau}}{\partial p_{\mu}^{\alpha}}\left(p_{\mu+1_{n}}^{\alpha}-D_{\mu} \Phi^{\alpha}\right)-\sum_{i=1}^{n-1}\left(M^{i}\right)_{\rho}^{\tau} D_{i} \Psi^{\rho}=0
$$

where $D_{i}$ denotes the formal derivative with respect to $x^{i}$.

Since (13) contains no derivatives with respect to $x^{n}$, we get for the combined system $\beta_{r}^{(n)}=m, \beta_{r}^{(n-k)}=m\left(\begin{array}{c}r+k-2 \\ r-2\end{array}\right)+\tilde{\beta}_{r}^{(n-k)}$. This yields the Cartan characters

$$
\alpha_{r}^{(n)}=0, \alpha_{r}^{(n-k)}=m\left(\begin{array}{c}
r+k-2 \\
r-1
\end{array}\right)-\tilde{\beta}_{r}^{(n-k)} .
$$


This generalizes the results of [6] to nonlinear systems and to constraints which depend on $x^{n}$ and which do not form a normal system themselves.

\section{Gauge Systems}

In gauge theories, determining the number of arbitrary functions in the general solution is not sufficient, because the arbitrariness stems partially from the symmetry. Really interesting is here the number of physical degrees of freedom. We showed already in [13], how it can be computed. Here we will give an improved version of these results and apply them to the analysis of gauge fixing conditions.

We consider gauge transformation of the form:

$$
\begin{gathered}
\bar{x}^{i}=\Omega^{i}\left(x^{j}\right), \\
\bar{u}^{\alpha}=\Lambda\left(x^{i}, u^{\beta}, \lambda_{a}^{(0)}(x), \partial \lambda_{a}^{(1)}(x), \ldots, \partial^{p} \lambda_{a}^{(p)}(x)\right)
\end{gathered}
$$

where $\gamma_{0}$ gauge functions $\lambda_{a}^{(0)}$ are entering algebraically, $\gamma_{1}$ gauge functions $\lambda_{a}^{(1)}$ are entering through their first derivatives etc.

We assume that all derivatives of order $l$ of the functions $\lambda^{(l)}$ are explicitly occuring in (17) and that all gauge functions depend on all independent variables. The only reason for these assumptions is simplicity. The formalism can handle more complicated situations, but the results become rather akward. Most gauge theories satisfy our assumptions anyway.

The symmetry (17) implies that

$$
G_{q}(r)=\sum_{l=0}^{p} \gamma_{l}\left[\begin{array}{c}
n \\
q+r+l
\end{array}\right]
$$

Taylor coefficients of order $q+r$ can be arbitrarily set by a gauge fixing. Thus they must be subtracted from the Hilbert polynomial to obtain the physical relevant number of free coefficients. We introduce the gauge corrected Hilbert polynomial

$$
\bar{H}_{q}(r)=H_{q}(r)-G_{q}(r) .
$$

Its coefficient of $r^{k}$ is given by

$$
\bar{h}_{k}=h_{k}-\frac{1}{(n-1) !} \sum_{l=0}^{p} \gamma_{l} s_{n-k-1}^{(n-1)}(q+l), \quad k=0, \ldots, n-1 .
$$

A closer look at (4) reveals that the coefficients of the Hilbert polynomial and the Cartan characters are in a one-to-one correspondance. Thus we can associate gauge corrected Cartan characters with $\bar{H}_{q}(r)$. They can be expressed in a recursion relation

$$
\bar{\alpha}_{q}^{(k)}=(k-1) ! \bar{h}_{k-1}-\sum_{i=k+1}^{n} \frac{(k-1) !}{(i-1) !} \bar{\alpha}_{q}^{(k)} s_{i-k}^{(i-1)}(0), \quad k=1, \ldots, n .
$$


To obtain now the the number of physical relevant free functions in the general solution of our systems, we simply apply the results of Section 3 to the gauge corrected Cartan characters and Hilbert polynomial, respectively. This yields for instance the following modification to Theorem 1.

Theorem 3 If the physical solution space has a representation satisfying the assumptions of Theorem 1, then it is spanned by $p_{k}$ arbitrary functions with $k$ arguments, where $p_{k}=f_{k}-g_{k}$ with

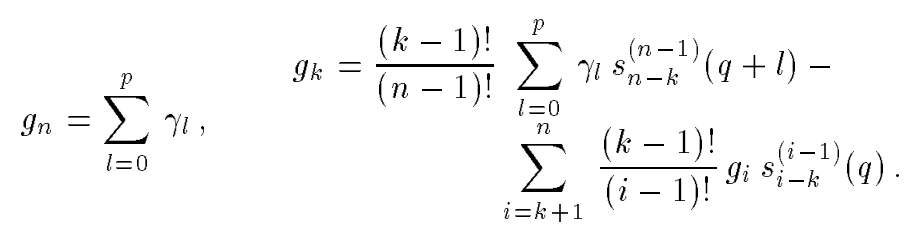

Similiarly, we obtain a correction for the strength of a gauge system

$$
\bar{Z}_{q}^{(1)}=(n-1)\left(\frac{1}{2} n \alpha_{q}^{(n)}+\alpha_{q}^{(n-1)}-\sum_{l=0}^{p}\left(\frac{1}{2} n+q+l\right) \gamma_{l}\right) .
$$

Finally, we can easily compute the remaining gauge freedom after some gauge conditions are imposed, if they take the form of differential equations. Then these equations are added to the original system. This yields in general an overdetermined system. It should be invariant only under a proper subgroup of the gauge group (It is, however, possible that new symmetries arise!).

A necessary condition for a complete gauge fixing is that the Cartan characters of this system are the same as the gauge corrected Cartan characters of the original system (one can of course also use the Hilbert polynomial). Note, however, that this is only a necessary criterium, as there may still remain a finite-dimensional symmetry depending on some arbitrary constants. This cannot be checked with the Cartan characters, as they are only related with the number of arbitrary functions.

\section{Yang-Mills Equations}

As first example, we consider the field equations of a Yang-Mills theory with a $d$ dimensional gauge group in an $n$-dimensional space-time. The dependent variables are the $m=d n$ components $A_{\mu}^{a}$ of the vector potential. In terms of the field strengths $F_{\mu \nu}^{a}=\partial_{\mu} A_{\nu}^{a}-\partial_{\nu} A_{\mu}^{a}+C_{b c}^{a} A_{\mu}^{b} A_{\nu}^{c}$ where $C_{b c}^{a}$ are the structure constants of the Lie algebra of the gauge group the $d n$ field equations are

$$
\partial_{\mu} F_{\mu \nu}^{a}+C_{b c}^{a} A_{\mu}^{b} F_{\mu \nu}^{c}=0, \quad\left\{\begin{array}{l}
a=1, \ldots, d, \\
\nu=1, \ldots, n .
\end{array}\right.
$$

One can easily check that they are involutive.

To compute the Cartan characters, we must analyse the symbol or the principal part of (24). It is given by $\partial_{\mu \mu} A_{\nu}^{a}-\partial_{\mu \nu} A_{\mu}^{a}$. Thus for $\nu \neq n$ we can always take $\partial_{n n} A_{\nu}^{a}$ as principal derivative. If however $\nu=n$, then this term disappears and we 
get $\partial_{n, n-1} A_{n-1}^{a}$ as principal derivative. This yields $\beta_{Y M}^{(n)}=d(n-1), \beta_{Y M}^{(n-1)}=d$. All other $\beta_{Y M}^{(k)}$ vanish. Thus the Yang-Mills equations do not form a normal system. For the Cartan characters we get

$$
\alpha_{Y M}^{(n)}=d, \alpha_{Y M}^{(n-1)}=d(2 n-1), \alpha_{Y M}^{(n-k)}=d n(k+1) .
$$

The field equations (24) are invariant under gauge transformations

$$
\bar{A}_{\mu}^{a}=A_{\mu}^{a}+\partial_{\mu} \lambda^{a}+C_{b c}^{a} A_{\mu}^{b} \lambda^{c},
$$

i.e. we have $\gamma_{1}=d$ gauge functions $\lambda^{a}$. This yields for the gauge corrected strength

$$
\bar{Z}_{Y M}^{(1)}=2(n-1)(n-2) d .
$$

For simplicity, we restrict ourselves now to $n=4$. (4) leads to the Hilbert polynomial

$$
H_{Y M}(r)=36 d+\frac{73}{3} d r+\frac{9}{2} d r^{2}+\frac{1}{6} d r^{3} .
$$

Adjusting for the gauge symmetry (26) yields the corrected polynomial

$$
\bar{H}_{Y M}(r)=16 d+12 d r+2 d r^{2}
$$

and the corrected Cartan characters

$$
\bar{\alpha}_{Y M}^{(4)}=0, \bar{\alpha}_{Y M}^{(3)}=4 d, \bar{\alpha}_{Y M}^{(2)}=\bar{\alpha}_{Y M}^{(1)}=6 d
$$

We see that the dimension $d$ of the gauge group appears everywhere linearly. Thus we get just $d$-times the values of the Maxwell equations. For them there exists also a first-order formulation using the field strengths as dependent variables. Since then only gauge invariant objects are used, the equations no longer exhibit the gauge symmetry (26) and lead directly to the Cartan characters (30) [13].

Finally, we note that the results are independent of the structure of the gauge algebra, as the structure constants do not appear in the symbol. Especially it makes no difference whether we treat an abelian or a non-abelian theory.

To conclude the analysis of the Yang-Mills equations, we consider as an example of a gauge fixing the Lorentz gauge $\partial_{\mu} A_{\mu}^{a}=0$. Adding this condition to the field equations (24) leads to a simple overdetermined system with the Cartan characters

$$
\alpha_{\text {Lor }}^{(4)}=0, \alpha_{\text {Lor }}^{(3)}=4 d, \alpha_{\text {Lor }}^{(2)}=12 d, \alpha_{\text {Lor }}^{(1)}=16 d
$$

A comparison with (30) shows that although the first two characters have the same values as the gauge corrected ones, the lower two are too large. Thus the Lorentz condition does not completely fix the gauge.

\section{Einstein's Vacuum Field Equations}

As second example, we treat Einstein's vaccuum field equations for an $n$-dimensional space-time

$$
R_{\mu \nu}=0
$$


If we take the components of the metric tensor $g_{\mu \nu}$ as dependent variables, we have because of the symmetries of Ricci tensor and metric, respectively, $n(n+1) / 2$ equations for as many dependent variables.

Expanding (32) in a general coordinate system in terms of $g_{\mu \nu}$ leads to very complicated expressions. It is, however, well-known, that one can find to any point $x_{0}$ of space-time a coordinate system such that in $x_{0}$ the Christoffel symbols vanish and $g_{\mu \nu}\left(x_{0}\right)=\eta_{\mu \nu}$, the constant metric of Minkowski space.

In these locally geodesic coordinates, (32) takes in $x_{0}$ the form [14]

$$
R_{\mu \nu}\left(x_{0}\right)=\frac{1}{2} \eta^{\rho \sigma}\left(\partial_{\mu \rho} g_{\nu \sigma}+\partial_{\nu \sigma} g_{\mu \rho}-\partial_{\mu \nu} g_{\sigma \rho}-\partial_{\sigma \rho} g_{\mu \nu}\right)=0
$$

In this form it is not difficult to show that the Einstein equations are involutive.

Because of the symmetry, we can assume $\mu \geq \nu$. We must distinguish four cases in the analysis of the symbol:

(i) $\mu \neq n$ and $\nu \neq n$ : The principal derivative is $\partial_{n n} g_{\mu \nu}$.

(ii) $\mu=n$ and $\nu<n-1$ : The principal derivative is $\partial_{n, n-1} g_{n-1, \nu}$.

(iii) $\mu=n$ and $\nu=n-1$ : The principal derivative is $\partial_{n-1, n-1} g_{11}$.

(iv) $\mu=n$ and $\nu=n$ : The principal derivative is $\partial_{n, n-1} g_{11}$.

Besides the last case this can be seen at once. For (iv) several substitutions of other equations are necessary.

This gives $\beta_{\text {Ein }}^{(n)}=n(n-1) / 2, \beta_{\text {Ein }}^{(n-1)}=n$. The other $\beta_{\text {Ein }}^{(k)}$ vanish. Thus the Einstein equations do not form a normal system. Their Cartan characters are

$$
\alpha_{\text {Ein }}^{(n)}=n, \alpha_{\text {Ein }}^{(n-1)}=n^{2}, \alpha_{\text {Ein }}^{(n-k)}=\frac{n(n+1)(k+1)}{2} .
$$

Since $x_{0}$ was an arbitrary point, these results remain valid on the entire space-time.

(32) is invariant under gauge transformations

$$
g_{\mu \nu}=\frac{\partial \bar{x}^{\rho}}{\partial x^{\mu}} \frac{\partial \bar{x}^{\sigma}}{\partial x^{\nu}} \bar{g}_{\rho \sigma}
$$

with $\gamma_{1}=n$ gauge functions $\bar{x}^{\mu}$. This yields a gauge corrected strength

$$
\bar{Z}_{\text {Ein }}^{(1)}=n(n-1)(n-3) \text {. }
$$

Analogously to our analysis of the Lorentz gauge, we can consider harmonic coordinates, i.e. we pose the gauge condition

$$
\square x^{\mu}=\frac{1}{\sqrt{-g}} \partial_{\nu}\left(\sqrt{-g} g^{\mu \nu}\right)=0 .
$$

In locally geodesic coordinates for $x_{0}$ this is equivalent to $\partial_{\nu} g_{\mu \nu}\left(x_{0}\right)=0$. After prolonging to second order, we get thus $n^{2}$ additional equations to (33). One sees easily that always $n$ of them are in the same class. Hence, the Cartan characters of the combined system are

$$
\alpha_{H}^{(n)}=0, \alpha_{H}^{(n-1)}=n(n-1), \alpha_{H}^{(n-k)}=\frac{n^{2}(k+1)+n(k-1)}{2} .
$$


Thus (37) provides no complete gauge fixing, because already $\alpha_{H}^{(n-1)}$ is too big, as we obtain for the highest gauge corrected Cartan characters

$$
\bar{\alpha}_{\text {Ein }}^{(n)}=0, \bar{\alpha}_{\operatorname{Ein}}^{(n-1)}=n(n-3) \text {. }
$$

Penney [9] and Mariwalla [5] computed already the strength of the Maxwell and Einstein equations in arbitrary dimensions using Einstein's approach of counting equations and identities. Penney overlooked, however, the existence of additional identities for the Maxwell equations in higher dimensions and got wrong results. This clearly indicates the advantage of the formal approach. The number of identities is encoded in the Cartan characters. Actually, it is even possible to compute it this way. Pommaret [11] calculated e.g. with formal methods the number of independent Bianchi identities for a Riemannian manifold of arbitrary dimension.

\section{Acknowledgements}

The author wishes to thank J.F. Pommaret for many helpful discussions and P. Winternitz for his hospitality at the CRM in Montréal where most of the here presented work was done. He is supported by Studienstiftung des deutschen Volkes.

\section{References}

1. G.W. Bluman, S. Kumei: Symmetries and Differential Equations. Applied Mathematical Sciences 81. Springer-Verlag, New York 1989.

2. A. Einstein: The Meaning of Relativity. Princeton University Press, Princeton, 5th edition, 1955.

3. M. Janet: Sur les Systèmes d'Équations aux Derivées Partielles. J. Math. 3(1920)65-151.

4. F. John: Partial Differential Equations. Applied Mathematical Sciences 1. Springer-Verlag, New York 1982.

5. K.H. Mariwalla: Applications of the Concept of Strength of a System of Partial Differential Equations. J. Math. Phys. 15 (1974) 468-473.

6. S.V. Meleshko: Differential Constraints and One-Parameter Lie-Bäcklund Transformation Groups. Sov. Math. Dokl. 28 (1983) 37-41.

7. P.J. Olver: Applications of Lie Groups to Differential Equations. Graduate Texts in Mathematics 108. Springer-Verlag, New York 1985.

8. P.J. Olver, P. Rosenau: Group-Invariant Solutions of Differential Equations. SIAM J. Appl. Math. 47 (1987) 263-278.

9. R. Penney: On the Dimensionality of the Real World. J. Math. Phys. 6 (1965) 1607-1611.

10. J.F. Pommaret: Systems of Partial Differential Equations and Lie Pseudogroups. Gordon\& Breach, London 1978.

11. J.F. Pommaret: Explicit Calculation of Certain Differential Identities used in Mathematical Physics. Proc. Differential Geometry and its Applications, Brno 1986, Math. Appl., East Eur. Ser. $27(1987) 271-278$.

12. J. Schü, W.M. Seiler, J. Calmet: Algorithmic Methods for Lie Pseudogroups. Proc. Modern Group Analysis, Acireale 1992. N.H. Ibragimov, M. Torrisi, A. Valenti (eds.). Kluwer, Dordrecht, to appear.

13. W.M. Seiler: On the Arbitrariness of the General Solution of an Involutive Partial Differential Equation. J. Math. Phys., to appear.

14. H. Stephani: Allgemeine Relativitätstheorie. Deutscher Verlag der Wissenschaften, Berlin 1980 . 